\title{
Super resolution for X-ray scattering and biological insights from its applications to dynamic DNA replication and repair complexes
}

\author{
John A. Tainer, ${ }^{a, b}$, Susan E. Tsutakawa ${ }^{a}$, Greg L. Huraa, Michal Hammela, \\ a Molecular Biophysics and Integrated Bioimaging, Lawrence Berkeley National Laboratory, Berkeley, \\ CA 94720, USA \\ ${ }^{\mathrm{b}}$ Department of Molecular and Cellular Oncology, The University of Texas M. D. Anderson Cancer \\ Center, Houston, TX 77030, USA
}

\begin{abstract}
Biological small angle X-ray scattering (BioSAXS) is an under appreciated and under utilized method for structural analyses that accuractely defines solution conformations and assemblies that are directly relevant to connecting structures to biological insights. Bio-SAXS is the only true high-throughput structural biology method. It furthermore provides information on the entire complex including both ordered and flexible regions when interpreted with appropriate metrics and methods. We will present data on dynamic complexes acting in DNA replication and repair. The results will show that SAXS can probe at resolutions sufficient to distinguish different conformational states, characterize flexible macromolecules, and screen in high-throughput under most solution conditions. Methods with exemplary experimental results will be considered with a focus on interpretation tools. Results will be presented to show how SAXS can provide accurate shapes, assembly states, and comprehensive conformations in solution that inform biology in fundamental ways ${ }^{1-9}$. The approaches for measuring flexibility, conformational changes, and assembly processes discussed here are relevant for accurate understanding, simulation, and prediction of many mechanisms in cell biology and nanotechnology.
\end{abstract}

1. Putnam, C.D., Hammel, M., Hura, G. L., and Tainer, J. A. (2007) X-ray solution scattering (SAXS) combined with crystallography and computation: defining accurate macromolecular structures, conformations and assemblies in solution, Quarterly Reviews in Biophysics 40, 191-285.

2. Rambo RP, Tainer JA. (2011) Characterizing flexible and intrinsically unstructured biological macromolecules by SAS using the Porod-Debye law. Biopolymers 95, 559-571. 13

3. Hura, G.L., Menon, A.L., Hammel, M., Rambo, R.P., Poole, F.L., Tsutakawa, S.E., Jenney, F.E., Frankel, K.A., Hopkins, R.C., Scott, J.O., Dillard, B.D., Classen, S., Adams, M.W.W. and Tainer, J.A. (2009) Rapid and robust proteomics-scale solution structural analyses determined efficiently by $\mathrm{X}$-ray scattering (SAXS) Nature Methods 6, 606-612.

4. Classen S, Hura GL, Holton JM, Rambo RP, Rodic I, McGuire PJ, Dyer K, Hammel M, Meigs G, Frankel KA, Tainer JA. (2013) Implementation and performance of SIBYLS: a dual endstation smallangle $\mathrm{X}$-ray scattering and macromolecular crystallography beamline at the Advanced Light Source. J Appl Crystallogr. 46,1-13.

5. Rambo RP and Tainer JA. (2013) Accurate assessment of mass, models and resolution by smallangle scattering of macromolecules, Nature 96, 477-481.

6. Hura, GL, Budworth, H., Dyer, KN, Rambo, R.P. Hammel, M., McMurray, CT., and Tainer JA. (2013) Comprehensive macromolecular conformations mapped by quantitative SAXS analyses, Nature Methods 10, 453-454.

7. Rambo RP and Tainer JA. (2013) Super-Resolution in Solution X-Ray Scattering and Its Applications to Structural Systems Biology, Annu. Rev. Biophys, 42, 415-441

8. Lai YT, Hura GL, Dyer KN, Tang HY, Tainer JA, Yeates TO. (2016) Sci Adv. 2, e1501855.

9. Wallen JR, Zhang H, Weis C, Cui W, Foster BM, Ho CM, Hammel M, Tainer JA, Gross ML, Ellenberger T. (2017) Hybrid Methods Reveal Multiple Flexibly Linked DNA Polymerases within the Bacteriophage T7 Replisome. Structure 25, 157-166. 